\title{
Beitrag der Geographie zu einer neuen Raumplanung
}

Unter diesen Titel stellte der Vorstand der Schweizerischen Gesellschaft für angewandte Geographie (SGAG/SSGA) die Jahrestagung 1985. Der Vorstand formulierte dazu vier Themenkreise und acht Prämissen, die eine möglichst angeregte Diskussion auslösen sollten. Es ist der Gesellschaft gelungen, Referenten zu finden, die die vorgegebenen Themenkreise engagiert vertraten. Auf dem Appenberg ob Zäziwil entwickelte sich in gelöster Atmosphäre eine fruchtbare Diskussion unter den zahlreichen Teilnehmern aus Forschung, Lehre und Praxis.

Die vier Themenkreise lauteten:

- Geographie in der Raumplanung

- Forschung, Praxis, Politik

- Transfer Wissenschaft/Raumplanung

- Ökologische Methoden in der Raumplanung

Die einzelnen Prämissen werden den Referaten vorangestellt.

\section{Geographie in der Raumplanung?}

\section{Die Geographie überläßt die Behandlung der raum- relevanten Problemstellungen zunehmend anderen Fachrichtungen}

Ulrich Roth:

Diese Frage Geographen vorzulegen scheint auf den ersten Blick ebenso absurd zu sein, wie einen Landwirt zu fragen, ob seine Tätigkeit etwas mit dem natürlichen Boden zu tun habe. Das ist doch selbstverständlich:

"Die Raumplanung Schweiz lebt in hohem Maße von den Dienstleistungen der Geographen sowie ihrer Beiträge an das wissenschaftliche Gespräch und die praktische Alltagsarbeit bei Behörden und privaten Planungsbüros.» (I)

Wenn gar ein Jurist die Rolle der Geographen in der Raumplanung so beurteilt, dürfen wir uns stolz in die Brust werfen!

Nun ist es ja eine Eigenart der Geographie, sich im allgemeinen und bezüglich Selbstverständnis im besonderen vornehmlich der Vergangenheit hinzuwenden. In diesem Zusammenhang sind natürlich Leistungen von Geographen in der Raumplanung mit Namen wie Gutersohn und Winkler eng verknüpft.
Wie steht es aber heute mit der Geographie in der Raumplanung? Geben wir dazu wiederum einem Nichtgeographen das Wort:

"Die Geographie hat in der Pionierphase der Schweizer Raumplanung an vorderster Front gestritten und Bedeutendes geleistet. Seither verdanken wir ihr immer wieder wichtige Beiträge. Allerdings, an der Weiterentwicklung der Raumplanung in der Schweiz war die Geographie recht zurückhaltend beteiligt, so jedenfalls der Eindruck eines Aussenstehenden.» (2)

Dieser Außenstehende, welcher sich so diplomatisch ausdrückt, muß es wissen, denn er ist immerhin Stellvertretender Direktor im Bundesamt für Raumplanung.

Eine Folgerung drängt sich daher auf:

- Die Geographie überläßt die Behandlung der raumrelevanten Problemstellungen zunehmend anderen Fachbereichen.

Ein paar Beispiele zur Untermauerung dieser Behauptung:

1. Die in der öffentlichen Diskussion heute oft verwendeten Angaben über den Kulturlandverlust (Stichwort $1 \mathrm{~m}^{2}$ pro Sekunde), welche aktueller denn je sind, wurden von einem Agronomen ermittelt und stammen aus dem Zeitraum von 1939 bis 1975. Sie sind von seiten der Geographie nie verifiziert, interpretiert, aktualisiert oder mit anderen Veränderungen in Beziehung gesetzt worden.

2. Hinter dem Bundesgesetz über die Raumplanung steht eine sachlich umfassende, geographisch anmutende Denkweise. Die Geographie hat dazu relativ wenig an Vorarbeiten beigetragen und soweit sie es überhaupt wahrgenommen hat wenig aus dem Gesetz gemacht, dies zumindest im Forschungsbereich.

Die gegenwärtig in Revision stehende Verordnung zu diesem Gesetz hat für die Entwicklung der Landschaft eine sehr große Bedeutung. Die einseitige Bevorzugung landwirtschaftlicher Interessen, welche zudem laut verschiedener Stellungnahmen zu diesem Entwurf nicht zulasten der Baugebiete gehen soll, ist augenfällig. Die Geographie war auch hier unseres Wissens nicht beteiligt.

3. Der Bundesrat hat - nicht zuletzt gestützt auf Vorarbeiten von seiten der Geographie - beschlossen, ein Nationales Forschungsprogramm «Nutzung des Bodens in der Schweiz» (wahrlich ein 
«geographischen» Titel) durchführen zu lassen. Die Geographie ist weder in der Expertengruppe noch in der Programmleitung vertreten. Spezialisten aus Landwirtschaft, Verkehrswesen, Recht und Ökonomie bestimmen nun über die Verwendung der Mittel.

4. Die Umweltverträglichkeitsprüfung ist ein neues Instrument, welches sehr nahe bei der Raumplanung einzustufen und von großer Relevanz für die Geographie ist. Methodische Vorarbeiten von seiten der Geographie sind dünn gesät. Ökonomen und Ingenieure haben sich hier aber bereits sehr stark engagiert.

Auch bei der Besetzung von Stellen, welche vom Anforderungsprofil her eindeutig geographische Tätigkeiten betreffen, halten vermehrt andere Berufsgattungen Einzug. Hier nur drei mir bekannte aktuelle Beispiele:

1. Die neugeschaffene Stelle eines Vizedirektors für den Bereich Landschaft im Bundesamt für Forstwesen und Landschaftsschutz wird mit einem Juristen besetzt.

2. Die Koordinationsstelle für Umweltschutz im Kanton Bern (zuständig insbesondere für Umweltverträglichkeitsprüfungen) hat neben ihrem Leiter, einem Juristen, soeben einen Ethologen angestellt.

3. Das Raumplanungsamt des Kantons Bern, welches neben dem zusätzlich vorhandenen Naturschutzinspektorat für die Landschaftsplanung zuständig ist, hat für diesen Bereich eine Stelle ausgeschrieben, welche nach unseren Informationen durch einen Zoologen besetzt werden soll.

Kann man den anderen Fachrichtungen einen Vorwurf machen, wenn sie sich ebenfalls um Lösungen aktueller räumlicher Probleme bemühen, welche von geographischer Seite nicht immer mit genügendem Nachdruck vertreten werden? Wo bleiben beispielsweise Beiträge der Geographie zu Themen wie

- Waldsterben

- Energie

- Verkehr

- Siedlungswesen

- methodische Lösungsansätze in der zeitlich dringlichen Nutzungsplanung?

Haben andere Fachrichtungen die Geographie entdeckt und versuchen, den Geographen ihr Feld streitig zu machen? Ich glaube, diese Betrachtungsweise ist zu einfach. Könnte es nicht auch so sein, daß die anderen Fachrichtungen im Zusammenhang mit aktuellen Problemstellungen aus ihrem Bereich den Kontakt zur Geographie suchen, ohne daß die Geographen dies wahrnehmen bzw. wahrnehmen wollen? Haben wir nicht oft das Gefühl, als einzige räumliche Problemzusammenhänge mindestens ahnen zu können?

Vielleicht müssen wir lernen, auch von der Geographie aus vermehrt interdisziplinär, d.h. mit den anderen Fachrichtungen zusammenzuarbeiten. Die anstehenden Probleme sind nicht mehr ausschließlich in fachspezifischen Gehegen zu bearbeiten, gerade deshalb sind sie aber auch geographische Probleme. Dabei erwartet man von der Geographie auch in politisch heiklen Situationen klare, sachlich begründbare Stellungnahmen und Argumente. Das MABProgramm hat meines Erachtens gezeigt, wo bescheidene Ansätze hiezu für die Geographie liegen könnten.

Ulrich Roth, dipl. Geograph ETH, Raumplaner Sigmaplan AG, Zähringerstr. 61, 3012 Bern

\section{Quellen:}

1) Lendi M., Raumplanung Schweiz - ein bleibender Auftrag, in: DISP Nr. 76, Institut für Orts-, Regional- und Landesplanung, ETH Zürich 1984

2) Flückiger $H$., Angewandte Geographie - Stellenwert in der Raumplanung, in: Geographica Helvetica 1984, Nr. 2

\section{Geographie in der Raumplanung? \\ Raumverständnis darf und kann weder bei den Behörden noch der Bevölkerung vorausgesetzt werden}

Hans Elsasser/Kurt Strub:

Das Raumverständnis bei Behörden und in der Bevölkerung ist nicht umfassend, sondern sektoriell. Der Raum wird als Naturraum, Schutzraum, Erholungsraum, Wirtschaftsraum usw. und nur selten in einem umfassenden Sinn als Lebensraum des Menschen betrachtet. Um Einzelinteressen besser durchsetzen zu können, wird teilweise bewußt, teilweise mangels fehlender Einsicht in die Zusammenhänge und Vernetzungen, auf eine gesamträumliche Sicht verzichtet.

Der Raumplaner sollte nun - unter Berücksichtigung der gesamträumlichen Zusammenhänge sowie der Beziehungen zwischen Gesamtraum und Teilräumen - diese verschiedenen Forderungen an den Raum gegeneinander abwägen und zu politisch tragfähigen Lösungen kommen. Im Gegensatz zu den Spezialisten fehlen aber dem Planer häufig wissenschaftliche und außerwirtschaftliche "pressure groups", um seine Anliegen, welche diejenigen des Gesamtraumes sind, durchzusetzen. Jedermann ist am Lebensraum interessiert, niemand setzt sich dafür ein; der Lebensraum besitzt keine Lobby.

Die Feststellung, daß zur Lösung heutiger und künftiger Probleme der räumlichen Ordnung und Organisation nicht nur Spezialisten, sondern auch Generalisten benötigt werden, ist weder neu noch originell.

Die in den letzten Jahren und Jahrzehnten erfolgte Spezialisierung innerhalb der geographischen Lehre 
und Forschung ist auch aus Sicht der Praxis zu begrüßen. Entschieden abgelehnt werden muß aber die Abschottung der einzelnen "Teilgeographien» innerhalb der Geographie.

Die raumplanerische Praxis verlangt Geographen, welche gleichzeitig über ein breites geographisches Wissen - sowohl in der physischen als auch in der Anthropogeographie - und über ein vertieftes, spezialisiertes Wissen in einzelnen "Teilgeographien» (inkl. den entsprechenden Nachbarwissenschaften) verfügen.

Vertieftes Wissen bedeutet ferner, daß der Geograph in der Lage ist, räumliche Probleme nicht nur zu beschreiben und zu erklären, sondern auch konkrete Lösungsvorschläge zu unterbreiten. Er muß mehr als Ideen liefern; er muß seine theoretischen und empirischen Untersuchungen so weit vorantreiben, daß die Realisierung der von ihm vorgeschlagenen Problemlösungen als gewährleistet angesehen werden darf.

Die Chancen des in der Raumplanungspraxis tätigen Geographen liegen eher in einem (auch methodisch) breiten als in einem hoch spezialisierten Wissen, welches ihm erlaubt, die Argumente der Spezialisten gegeneinander abzuwägen, zu gewichten und aus gesamträumlicher Sicht realistische Lösungsvorschläge zu unterbreiten. Gleichzeitig muß aber der Geograph mindestens in einem Teilgebiet über Spezialkenntnisse verfügen.

Bei diesem neuen "Geographen-Generalisten» handelt es sich um einen "Spezialisten für gesamträumliche Fragen».

Generalisten leben gefährlich: Die Grenze zwischen dem Generalisten und dem "Generaldilettanten" ist schmal. Es ist deshalb besonders wichtig, daß der Generalist nicht nur seine Möglichkeiten und Chancen, sondern vor allem auch seine Grenzen und Risiken deutlich erkennt. Generalisten haben auch größere Mühe, sich nach außen zu profilieren. Sie können nicht mit publikumswirksamen Gegensatzpaaren holzschnittartig argumentieren. Für die anstehenden Probleme unseres Lebensraumes werden aber nicht nur Schwarz-Weiss-Vorschläge benötigt; ebenso wichtig sind die Grautöne.

Die Zweiteilung des Geographiestudiums, "Grundstudium - Fachstudium", wie es verschiedene Hochschulen kennen, sollte durch eine Dreiteilung «Grundstudium - Fachstudium - integratives Geographiestudium» abgelöst werden. In diesem letzten Drittel würden - wenn möglich in Gruppenarbeiten komplexe gesamträumliche Probleme, d. h. Probleme, welche verschiedene geographische Spezialisierungsrichtungen betreffen, bis hin zu konkreten Lösungsvorschlägen bearbeitet; dadurch kann auch das Management einfacherer und komplexerer Projekte geübt werden. Im Rahmen von geographischen Diplomarbeiten und Disserationen sollten vermehrt als bisher geographische Teildisziplinen überschreitende Themen behandelt werden. Geographische Forschungsarbeiten sollten vermehrt im Grenzbereich zwischen physischer Geographie und Anthropogeographie angesiedelt werden. Generell: Die Interdisziplinarität innerhalb der Geographie - in Lehre und Forschung - muß verstärkt gepflegt werden.

Interdisziplinarität innerhalb der Geographie darf aber nicht zu einer Abkapselung der Geographie nach außen führen. Die Kontakte zu und die Konkurrenz mit anderen Wissenschaften sind für die Geographie lebenswichtig. Die Geographie ist nicht die einzige Wissenschaft, welche sich mit Problemen der räumlichen Ordnung und Organisation auseinandersetzt. Die Geographie muß über ihre angestammten Grenzen hinaus nachhaltige Kontakte pflegen (z. B. zu den Ingenieur- und Rechtswissenschaften).

Dieses neue (Berufs-)Bild des Geographen als Generalisten oder - was vermutlich publikumswirksamer ist - als Spezialisten für gesamträumliche Fragen muß nach außen bekannt gemacht werden. Grundvoraussetzung ist allerdings, daß zwischen Bild und Wirklichkeit keine Diskrepanz besteht. Dies ist eine Aufgabe der SGAG.

Im weitern hat sich die SGAG den Problemen der Fort- und Weiterbildung, der «éducation permanente», der Spezialisten für gesamträumliche Fragen anzunehmen. Dies hat in enger Zusammenarbeit mit den Hochschulen, der Praxis und nicht zuletzt mit anderen Berufsorganisationen zu geschehen. Gerade der Generalist ist gezwungen, sich intensiv und breit weiterzubilden, damit er Generalist bleibt und nicht zum Generaldilettanten verkommt.

Hans Elsasser, Prof. Dr., ORL-Institut, ETH Zürich, 8093 Zürich Kurt Strub, dipl. Geograph, Planer BSP, Stierli \& Partner AG, Büchnerstr. 26, 8006 Zürich

\section{Forschung, Praxis, Politik}

Die Geographie unterstützt den politischen Auftrag, wie er als Anliegen der Umweltgesetzgebung formuliert ist, zu wenig

Jürg Rohner:

\section{Vorbemerkung}

Im folgenden wird zu der von den Veranstaltern vorgegebenen These aus der Sicht eines in der Naturund Landschaftsschutzpraxis tätigen Geographen Stellung genommen. Dabei sollen einige Einschränkungen gelten:

- Die Beispiele sind alle der Bundesgesetzgebung entnommen, auf die umfangreiche kantonale $\mathrm{Ge}$ setzgebung kann nicht näher eingegangen werden. 
- Der politische Auftrag der Umweltgesetzgebung wird nur insofern skizziert, als er Berührungspunkte mit der Geographie aufweist.

\section{Der politische Auftrag der Umweltgesetzgebung}

Eine große Zahl eidgenössischer Gesetze enthält (meist randlich) einzelne Bestimmungen über die Behandlung der Umwelt. Als Beispiel sei Art.79, Abs. 1 des Bundesgesetzes (BG) über die Förderung der Landwirtschaft und die Erhaltung des Bauernstandes unter dem Titel «Bodenverbesserungen» erwähnt: «Den allgemeinen Interessen der Umwelt, insbesondere der Erhaltung des Grundwassers und der damit verbundenen Trinkwasserversorgung sowie dem Schutze der Natur und der Wahrung des Landschaftsbildes ist Rechnung zu tragen.»

In einem engeren Sinne können wir folgende Gesetze zur Umweltgesetzgebung der Schweiz zählen:

- BG betreffend die eidgenössische Oberaufsicht über die Forstpolizei vom 11. Oktober 1902 (FPolG)

- BG über den Natur- und Heimatschutz vom 1. Juli 1966 (NHG)

- BG über den Schutz der Gewässer gegen Verunreinigung vom 8. Oktober 1971 (GSchG)

- BG über die Raumplanung vom 22. Juni 1979 (RPG)

- BG über den Umweltschutz vom 7.Oktober 1983 (USG)

Alle diese Gesetze haben zum Ziel, die natürlichen Lebensgrundlagen zu erhalten, Eingriffe des Menschen in die Umwelt gewissen Regeln zu unterstellen und eingetretene Schäden soweit möglich zu beheben.

Führen wir uns kurz einige Zweckartikel zur Erinnerung vor Augen.

Art. 1 NHG:

«Dieses Gesetz hat zum Zwecke...,

A) das heimatliche Landschafts- und Ortsbild, die geschichtlichen Stätten sowie die Natur- und Kulturdenkmäler des Landes zu schonen; ...»

Art. 1, Abs. 1 USG:

«Dieses Gesetz soll Menschen, Tiere und Pflanzen, ihre Lebensgemeinschaften und Lebensräume gegen schädliche oder lästige Einwirkungen schützen und die Fruchtbarkeit des Bodens erhalten.»

Art. 1, Abs. 1 RPG:

«Bund, Kantone und Gemeinden sorgen dafür, daß der Boden haushälterisch genutzt wird. Sie stimmen ihre raumwirksamen Tätigkeiten aufeinander ab und verwirklichen eine auf die erwünschte Entwicklung des Landes ausgerichtete Ordnung der Besiedlung. Sie achten dabei auf die natürlichen Gegebenheiten sowie auf die Bedürfnisse von Bevölkerung und Wirtschaft.»
Aus diesen Zielsetzungen lassen sich unschwer die Beziehungen zur Geographie herstellen. Von Landschaft ist die Rede, von haushälterischer Nutzung des Bodens, von Rücksicht auf die natürlichen Gegebenheiten und auch von der Erhaltung der Bodenfruchtbarkeit. Man sollte meinen, die Geographie werde durch diese Thematik direkt angesprochen. Es läßt sich nicht vermeiden, daß der politische Auftrag, wie er in den zitierten Gesetzesartikeln zum Ausdruck kommt, häufig unklar, ja sogar widersprüchlich formuliert ist. Das sollte aber Geographen (und natürlich auch andere Wissenschafter!) nicht davon abhalten, den ihnen zugespielten Ball aufzunehmen und aus ihrer Sicht einen Beitrag zur Lösung der gestellten Aufgaben zu leisten. Damit habe ich indirekt schon angedeutet, $\mathrm{da} \beta$ ich die bis heute geleisteten Beiträge noch als ungenügend betrachte, mit anderen Worten: daß ich der mir vorgegebenen These einige Berechtigung zuerkenne.

\section{Die schweizerische Geographie vor dem umweltpolitischen Auftrag}

Zur Klarheit sei festgehalten, was ich verstehe, wenn in der These steht: «Die Geographie unterstützt... zu wenig.» Es gibt zum Glück eine wachsende Zahl von Geographen, die in ihrem beruflichen Alltag sehr stark für die Ausführung der erwähnten umweltpolitischen Aufträge tätig sind. Die These kann also nicht "die Geographen» schlechthin meinen, sondern vielmehr die institutionalisierte Geographie, worunter ich einerseits Forschung und Lehre an den Hochschulen, andererseits die Interessenvertretung durch Fachgesellschaften und Fachkommissionen verstehe.

Im Sinne von Beispielen will ich kurz einige Fragestellungen andeuten, die für die Geographie von Bedeutung sein müßten.

Das Raumplanungsgesetz verlangt eine «haushälterische Nutzung des Bodens». Um dieser Forderung nachzukommen, wäre es u. a. dringend nötig, mehr über die Verträglichkeit von sich überlagernden Nutzungen zu wissen. Ich betrachte es als eines der Grundübel unserer Raumplanung, daß wir für jeden Zweck eine besondere Zone reservieren wollen und so bei der Beschränktheit unseres Bodens fast von selbst zu Nutzungskonflikten und "Landschaftsverbrauch» kommen. Ich erwähne die mangelnde Überlagerung von Siedlung und Naherholung als Beispiel. Oder den ganz banalen Fall, um zu zeigen, was ich meine: Wie viele Hektaren Fläche werden während höchstens vier Monaten als Gartenbad genutzt und bleiben den Rest des Jahres eingehagt ohne Nutzung? Sind wir nicht in der Raumplanung bis jetzt zu phantasielos?

Ein zweites Beispiel. Es läßt sich wohl kaum bestreiten, daß der Bau von Verkehrswegen unser Land in den letzten zweieinhalb Jahrzehnten in seiner räumlichen Entwicklung direkt und vor allem indirekt sehr 
stark beeinflußt hat. Unter den direkten Auswirkungen wäre etwa der sogenannte Zerschneidungseffekt zu nennen. Kriterien zur diesbezüglichen Beurteilung von Projekten für neue Verkehrswege (neben Straßen ist auch der Neubau von Eisenbahnlinien aktuell) fehlen für die Verhältnisse in unserem Land weitgehend. $\mathrm{Zu}$ den indirekten Auswirkungen neuer Anlagen der Verkehrsinfrastruktur gehören u. a. die Veränderungen in der Siedlungsstruktur und im Freizeitverhalten. Die meisten wissenschaftlichen Untersuchungen beschränken sich darauf, im nachhinein festzustellen, was sich verändert hat. Selten sind die Versuche, aus bisherigen Entwicklungen Lehren zu ziehen und auf neue Projekte zu übertragen, um zu einer umfassenden Beurteilung zu kommen.

Zum Wahrnehmen des politischen Auftrages gehört neben Forschung und Lehre auch der Kontakt mit den Organen, die für den Vollzug der Umweltgesetzgebung zuständig sind. Auf kantonaler Ebene gibt es einige gute Beispiele, gesamthaft gesehen sind die Geographen in den Ämtern und Kommissionen aber noch ungenügend präsent. Auf eidgenössischer Ebene ist der Kontakt zur Vollzugspraxis ebenfalls unterschiedlich gut entwickelt. So wäre etwa darauf hinzuweisen, daß im sogenannten Forschungsstab des Bundesamtes für Raumplanung kein Geograph sitzt.

\section{Wünsche des Praktikers}

Der Praktiker, der im Bereich des Vollzugs der Umweltgesetzgebung tätig ist, hat verständlicherweise den Wunsch nach «Rezepten». Im Alltag mit seinen Hunderten von einzelnen Geschäften ist es nicht möglich, bei der Behandlung eines bestimmten Falles zuerst einige Dissertationen zu lesen und dann deren Ergebnisse umzusetzen. Häufig lassen das schon die Termine nicht zu. So muß etwa eine Beschwerde an das Bundesgericht betreffend Festlegung einer Restwassermenge oder an den Bundesrat betreffend Konzession einer touristischen Erschließung innert dreißig Tagen eingereicht sein. Der Praktiker ist deshalb darauf angewiesen, daß ihm der Forscher mehr entgegenkommt als heute und bereits einen Teil der Umsetzungsarbeit leistet.

In die gleiche Richtung zielt der Wunsch nach einfach reproduzierbaren Methoden. Lieber ein suboptimales Resultat, das auf vernünftigen Abschätzungen beruht, als gar kein Resultat, das ist (leider) die Erfordernis der Praxis, so unwissenschaftlich das tönt.

\section{Schlu $\beta$}

Auf Grund meiner notgedrungen knappen Überlegungen komme ich zum Schluß, daß die mir zum Kommentar anvertraute These stimmt.

Jürg Rohner, Dr. phil., Geograph, Schweiz. Bund für Naturschutz, 4020 Basel

\section{Die Forschung zum Landschaftswandel muß vertieft und koordiniert werden und darf sich nicht auf Vorher-/Nachher-Analysen beschränken}

\author{
Charles Mäder:
}

\section{Problemstellung}

Die Veränderungen der Landschaft werden vom Autor vor allem aus seiner beruflichen Tätigkeit als Geschäftsführer der Region Burgdorf und der Bergregion Trachselwald betrachtet. Der noch vorwiegend ländliche Raum des unteren und mittleren Emmentals, der allerdings in Teilen bereits unter den Druck aus der Agglomeration Bern gerät, bietet einem Geographen als Raumplaner ein besonders interessantes Beobachtungsfeld. Allerdings können Veränderungen wegen der starken Arbeitsbelastung meist nur festgestellt werden, zu einer tieferen und nötigen Analyse fehlt aber die Zeit zum gründlichen Überdenken. Hier ist der Praktiker auf die Mithilfe der Wissenschaft angewiesen.

Bei der Beobachtung von Veränderungen überwiegt der optische Aspekt sehr stark, wahrgenommen wird vor allem, was ins Auge fällt. Andere wenig sichtbare oder langsam ablaufende Veränderungen, die von ebenso großer Bedeutung sein können, fallen kaum auf. $\mathrm{Zu}$ nennen sind hier vor allem Vorgänge in der Landwirtschaft und in der Ökologie. Um auch diese Veränderungen in seine Überlegungen einbeziehen zu können, ist der praktisch tätige Geograph in diesem Bereich besonders auf die Unterstützung durch die Wissenschaft angewiesen.

Die Veränderungen, die in der Landschaft sichtbar werden, sind immer nur der letzte Schritt von Wirkungsketten. Es hilft dem Planer herzlich wenig, sich noch aufzuregen, wenn Bauprofile auf einer wertvollen Ackerfläche stehen oder wenn auf einem Hof die Gant angesagt ist. Der Planer kann seine Tätigkeit nur erfolgreich gestalten, wenn er allen anderen, in einem bestimmten Raum handelnden Personen um die berühmte Nasenlänge voraus ist, sowohl in seinen Kenntnissen der Grundlagen und Zusammenhänge als auch in seinem Informationsstand über den Ablauf der verschiedensten Vorgänge. In unserer auf individuellen Entscheiden sehr föderalistisch aufgebauten Gesellschaft ist allein schon die Informationsbeschaffung eine schwierige Aufgabe, besonders weil die Regionalplanung im Entscheidungsweg kaum eingebaut ist.

Aus der geschilderten Situation ergeben sich Forderungen an die angewandte Geographie als Forschungszweig:

- Sie muß sich auch mit wenig spektakulären Veränderungen befassen und sie untersuchen.

- Sie darf nicht nur einen Zustand vor und nach der Veränderung beschreiben, das Hauptgewicht muß auf der Erforschung der Wirkungsketten liegen. 
- Sie muß rasch zu Resultaten kommen und darf sich vor spekulativen Gedanken nicht scheuen.

- Sie muß ihre Resultate einfach, kurz und lesbar präsentieren.

- Sie muß glaubwürdig sein.

\section{Zusammenhänge, Grundlagen und Vorgehen}

Aus der Sicht einer Region ist es natürlich ideal, wenn gerade ihr Raum für eine Forschungsarbeit ausgewählt wird. Es ist aus dieser Sicht zu hoffen, daß z. B. die MAB-Testgebiete die Konsequenzen aus den Arbeiten ziehen werden, obwohl bezweifelt werden muß, daß die Erkenntnisse und Folgerungen bis zum einzelnen Stimmbürger hinunter bekannt geworden sind und damit Einfluß auf die letztlich immer politischen Entscheide nehmen können.

Ist eine Region nicht typisch oder sind ihre Veränderungen wenig spektakulär, so vermag sie das Interesse der Forscher kaum auf sich zu lenken. Was nützt in einem normalen Dorf die beste Analyse der Vorgänge bei einem Autobahnkreuz, wenn es langsam durch das Einsickern von stolzen Einfamilienhausbauherren in seiner sozialen und kulturellen Substanz Schaden erleidet? In diesen Räumen solle der regionale Planer möglichst alle Forschungsergebnisse verfolgen können, die Resultate auf die Brauchbarkeit im eigenen Raum prüfen und entsprechend anpassen können. Aber welcher Praktiker kann diese Arbeit selber leisten? Es ist deshalb zu fordern, daß alle raumwirksamen Arbeiten mindestens einen Abriß über die Grundzüge und deren Anwendungsbereich enthalten und Zusammenhänge und Wirkungsketten klar aufgezeigt werden.

Forscher jeder Stufe stellen sich ihre Fragen oft ohne vorherigen Kontakt mit den Praktikern. So entstehen zwar gründliche und gute Arbeiten, die vom Praktiker aber noch der "Übersetzung» bedürfen oder die an den Problemen vorbeigehen. Es ist deshalb auch zu fordern, daß Forscher in angewandter Geographie den Kontakt mit Kollegen, die in der Praxis eines Gebietes tätig sind, suchen, bevor sie Inhalt und Wege ihrer Arbeit festlegen. Der Praktiker will sich damit bestimmt nicht zum Vormund des Forschers machen, aber es gilt immer zu bedenken, daß Forschung in unserem Fachgebiet mit öffentlichen Geldern betrieben wird und daß der Bürger und Steuerzahler einen Anspruch darauf hat, Ergebnisse zu erhalten, die verwendet werden können.

\section{Vorher-/Nachher-Analysen}

Es ist gängige Praxis, zuerst aus den Quellen einen früheren Zustand zu rekonstruieren und anschließend eine bestehende Situation aufzunehmen und die Differenzen festzustellen. Diese Vorher-/Nachher-
Bild ist ja, was dem Betrachter selbst auffällt, seine Frage gilt aber dem Wie und Warum. Gegen das Vorgehen an sich sind keine Einwände zu erheben, wenn sich Bearbeiter immer bewußt sind, daß in der Realität die untersuchten Vorgänge kontinuierlich ablaufen und sich ständig ändern, auch über die Untersuchung hinaus. Dem Praktiker dienen Erkenntnisse über Vorgänge an sich, über Ursachen und Wirkungen, viel mehr als eine möglichst genaue Aufnahme eines Zustandes. Die Erfahrung lehrt, daß sich grundlegende Ursachen und Wirkungen über die Zeit wenig ändern, die «Suchb) nach den neusten statistischen Zahlen also sinnlos ist und kaum hilft.

\section{Der Zeitfaktor und der spekulative Gedanke}

Es ist verständlich, daß der Wissenschafter sich nicht drängen lassen will, daß er seine Erkenntnisse breit abstützen und verifizieren muß. In der Praxis spielt der Zeitfaktor aber eine große, oft entscheidende Rolle. Was nützt es dem Praktiker, wenn er drei Jahre auf Zählergebnisse und acht Jahre auf Analysen warten muß. In der Zwischenzeit fallen Entscheide, die wiederum erst in einer nächsten Periode erfaßt werden können. Nicht die letztmögliche Genauigkeit ist wichtig, sondern das rechtzeitige Vorliegen von Resultaten und Methoden, die es erlauben, ein anstehendes Problem zu lösen. Noch wertvoller sind natürlich diejenigen Arbeiten, die einen Problemkreis angehen, bevor er akut wird. Der Forscher sollte deshalb ein Sensorium für kommende Fragen haben. Es ist klar, daß dies meist ein Wunsch bleiben muß. Forscher orientieren sich vielfach nur an den Bedingungen einer wissenschaftlichen Arbeitsweise und scheuen sich, unbelegte oder intuitive Gedanken in ihre Arbeit einfließen zu lassen. Dazu kann nur bemerkt werden, daß nach einer gewissen Zeit niemand mehr sich um die Genauigkeit einer Statistik kümmert, sondern nur noch der Kerngedanke einer Arbeit Bestand hat. Der Grundwert einer Arbeit besteht in ihren neuen Gedanken. Beim Lesen von Fachliteratur wähnt man sich leider zu oft unter Wiederkäuern.

\section{Präsentation und Glaubwürdigkeit}

Ein Planer in der Praxis findet keine Zeit, langatmige Berichte zu lesen. Er kann es seinen Partnern in Gemeinden und anderen Gremien (meist Laien, die ihre Freizeit opfern) nicht zumuten, Fachchinesisch zu kennen und zu lesen. Der echte Wissenschafter zeichnet sich dadurch aus, daß er auch komplizierte Vorgänge anschaulich und verständlich darlegen kann und sich in Gebrauch von Fachausdrücken möglichst zurückhält. Sollte seine Arbeit intern an Instituten deswegen als zu banal bewertet werden, so kann hier 
nur gesagt werden, daß dann der Fehler viel eher im Institut als beim Autor zu suchen ist. Wir müssen uns beim sprachlichen Ausdruck unbedingt der angelsächsischen Schule zuwenden und unsere auf Deutschland ausgerichtete Tradition ablegen. Zum Umfang der Arbeiten nur soviel: Das Gewicht hat keinen Einfluß auf die Qualität.

Von Geographen ist zu fordern, daß gerade sie sich persönlich entsprechend der erteilten Ratschläge verhalten, sonst werden sie besonders im ländlichen Raum unglaubwürdig. Als Beispiel sei hier das Lamentieren über die Folgen der Zunahme der Pendler und der damit verbundenen Probleme genannt, das kaum verstanden wird, wenn sich herausstellt, daß der Bearbeiter ebenfalls seinen Wohnsitz weit weg von seiner Arbeitsstätte gewählt hat.

Der Praktiker ist darauf angewiesen, daß auch die wenig spektakulären Vorgänge durch die Wissenschaft untersucht werden, da $\beta$ ihm Resultate in kurzer verständlicher Form rasch zur Verfügung gestellt werden. Sind diese Voraussetzungen erfüllt, so ist Gewähr geboten für eine Zusammenarbeit, die für beide Seiten sehr fruchtbar sein kann.

Charles Mäder, Dr. phil. nat., Geograph, Planungsverband Region Burgdorf, 3400 Burgdorf

\section{Transfer Wissenschaft/Raumplanung}

\section{Die Raumplaner bemühen sich zuwenig darum, neue Erkenntnisse aus der Wissenschaft zur Kenntnis zu nehmen}

Urs Wiesmann:

Kommunikationsbarriere im Transfer zwischen geographischer Forschung und Raumplanung

\section{Einleitung und thematische Eingrenzung}

Als Restposten einer Thesenliste zur SGAG-Jahrestagung 1985 lag dem vorliegenden Beitrag die These, $\mathrm{da} ß$ sich die Raumplaner zuwenig darum bemühen, neue Erkenntnisse aus der Wissenschaft zur Kenntnis zu nehmen, zugrunde. Die Motivation, diese These zu vertreten, entsprang dem Bedürfnis, das Vorwurfsgefälle im unumstritten stockenden Kommunikationsfluß zwischen Forschung und Praxis (vgl. u.a. GH 2/84) etwas auszugleichen. Der Versuch, die genannte These zu begründen, mußte aber fallengelassen werden, und zwar nicht nur aufgrund des geringen Erfahrungspotentials des Autors, sondern auch aufgrund der Überlegung, da $ß$ die Diskussion der "Schuldfrage» in einem durch beide Partner gestalteten Kommunikationsumfeld kein sehr fruchtbarer Lösungsbeitrag sein kann.

Im vorliegenden Beitrag wird deshalb nicht die oben zitierte These diskutiert, sondern es sollen einige Überlegungen zu den primären Kommunikationsbarrieren zwischen den beiden Partnern angestellt werden, um davon ausgehend einige Ansätze zur Verbesserung des Kommunikationsflusses andeuten zu können. Die angetönte Thematik wird einschränkend nur im Zusammenhang mit der direkten Kommunikation zwischen wissenschaftlicher Forschung und Raumplanung diskutiert, d. h. Überlegungen zur Quantität und Qualität der zu transferierenden wissenschaftlichen Produkte und zu der sich über die Lehre abwickelnden indirekten Kommunikation werden ausgeklammert.

Die folgenden Ausführungen müssen sehr kurz gehalten werden und erhalten damit Thesencharakter.

\section{Deckungsgrad inhaltlicher Anspruchs- und Anforderungsprioritäten}

Die geographische Wissenschaft bearbeitet verschiedene Typen von Inhalten, die stark vereinfacht in folgenden Kategorien zusammengefaßt werden können (vgl. Figur): Dokumentarische Inhalte (Analytische Daten und systematische Inventare), erklärende Inhalte (Theorien zu räumlich-sachlichen Prozessen und zusammenfassende Raumentwicklungstheorien) sowie bewertende Inhalte (sektorale Bewertungsmethoden und übergreifende Raumentwicklungskonzepte). Prinzipiell setzt die Geographie - allenfalls im Gegensatz zu ihren einzelnen Teildisziplinen (?) - die höchsten Anspruchsprioritäten im Bereich integraler Raumbetrachtungen, also bei den genannten inhaltlichen Kategorien der Raumentwicklungstheorien und Raumentwicklungskonzepte.

Die Anforderungsprioritäten der Raumplanung an die Wissenschaft decken sich mindestens auf der höchsten Prioritätsstufe mit den Anspruchsprioritäten der geographischen Forschung. Mit diesem hohen Dekkungsgrad der Anspruchs- und Anforderungsprioritäten drängt sich ein Transfer zwischen geographischer Forschung und Raumplanung geradezu auf.

Auch unter der Annahme, daß Anspruch und Realisierung in der Forschung übereinstimmen würden, kann der sich aufdrängende Transfer zwischen geographischer Forschung und Raumplanung nur beschränkt funktionieren, da sich die beiden Partner mindestens auf folgenden fünf Ebenen teilweise verpassen, wobei die daraus resultierenden Kommunikationsbarrieren in unterschiedlichem Maß den Transfer der oben genannten Kategorien von Inhalten erschweren: 


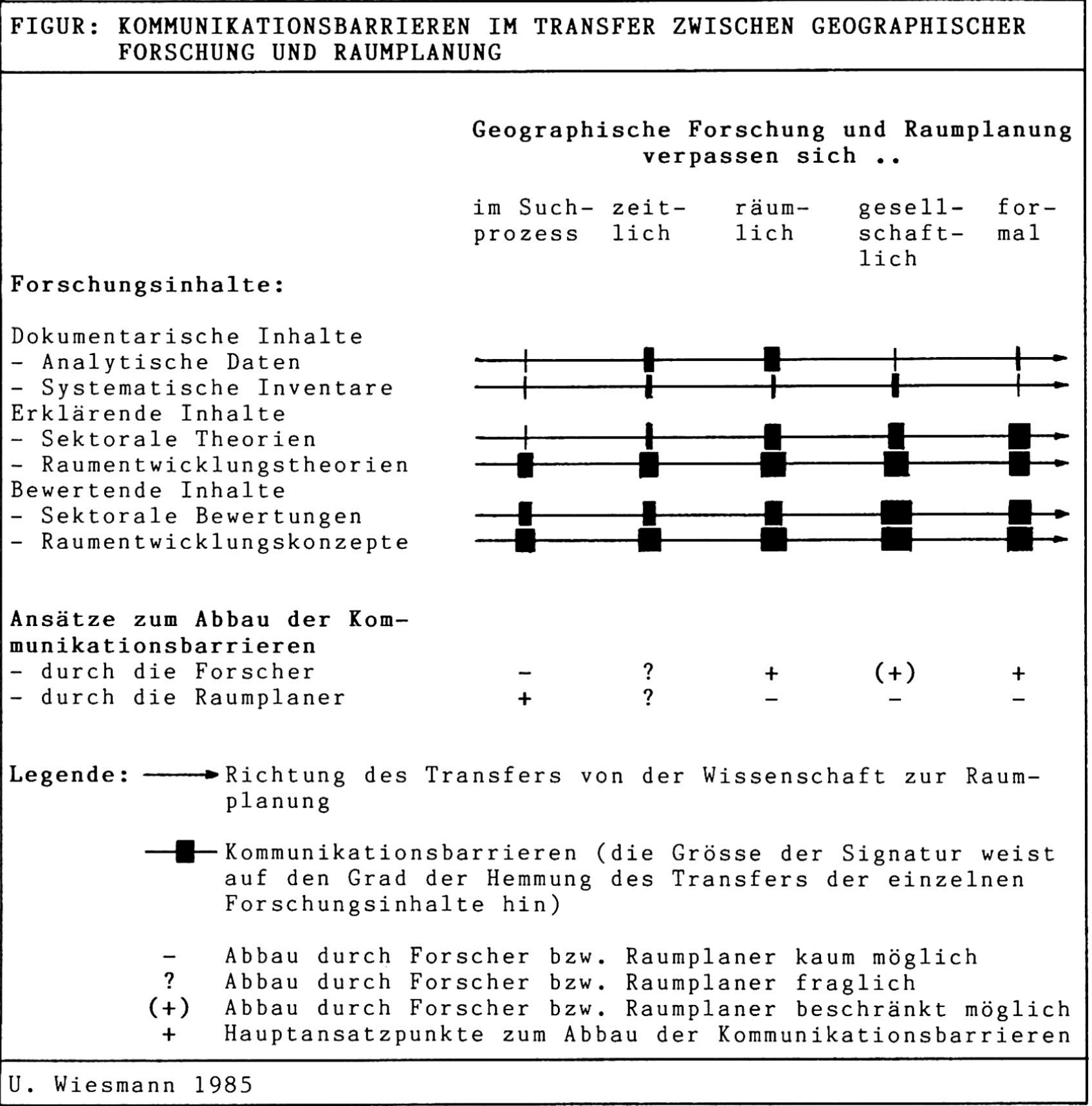

\section{Fünf Ebenen, auf denen sich Forschung und Raumplanung verpassen}

\section{a. Geographische Forschung und Raumplanung} verpassen sich am Suchprozeß:

Der Suchprozeß zur Erkenntnisgewinnung in der Forschung läuft, bezogen auf die genannten Kategorien von Inhalten, in umgekehrter Richtung als der Suchprozeß der Raumplanung nach transferierbaren wissenschaftlichen Inhalten: In einem iterativen Verfahren, das von dokumentarischen über erklärende zu bewertenden (und allenfalls zu operationellen) Inhalten führt, wird in der Forschung versucht, die genannten Anspruchsprioritäten zu erfüllen. Die Raumpla- nung beginnt ihren Suchprozeß hingegen auf der Ebene ihrer höchsten Anforderungsprioritäten, findet dort wenig und, durch das iterative Forschungsverfahren bedingt, nur Unfertiges, worauf sie ihre Anforderungen an die Wissenschaft sukzessive reduziert und schlußendlich bei der Mindestanforderung nach dokumentarischen Inhalten in Form systematischer Inventare stehenbleibt. Doch gerade diese Inventare nehmen im Anspruchsprofil der Forschung einen geringen Stellenwert ein, womit auch diese Mindestanforderung kaum befriedigend erfüllt werden kann. Aus der gegenläufigen Richtung der Suchprozesse von Forschung und Raumplanung ergibt sich also die unbefriedigende Situation, daß sich die beiden Part- 
ner, trotz des hohen Deckungsgrades von Anspruchsund Anforderungsprioritäten, höchstens auf der Ebene tiefer Anspruchs- und Anforderungsprioritäten treffen. Über die daraus resultierende Enttäuschung baut sich eine schwer auflösbare, gegenseitige Kommunikationshemmung auf.

\section{b. ...verpassen sich zeitlich:}

Wenn der Forschungsproze $\beta$ im Hinblick auf die Anspruchsprioritäten aufrechterhalten werden soll, hat jede konkrete Untersuchung Beispielcharakter, d. h. sie steht in einem grösseren Forschungskontext, der auch bei den zeitlich schnell wechselnden Anforderungen der aktuellen Politik nicht verlassen werden darf. Das zeitliche Zusammentreffen von konkreten wissenschaftlichen Untersuchungen und politischer Aktualität ist damit meist eher zufällig, insbesondere bei komplexeren Forschungsinhalten und damit bei den höheren Anspruchs- und Anforderungsprioritäten.

Hierzu kommt weiter, daß die schnell wechselnden tagespolitischen Aufgaben und Anforderungen es dem Raumplaner verunmöglichen, längerfristige Forschungsvorhaben kontinuierlich zu verfolgen, womit die Chance, daß eine Informationskampagne zu wissenschaftlichen Ergebnissen zeitlich unpassend angesetzt wird, sehr groß ist.

\section{c. ...verpassen sich räumlich}

Aus den gleichen Gründen, aufgrund deren sich Forschung und Praxis zeitlich verpassen, ist die Chance sehr groß, daß sich die Bearbeitungsräume von Wissenschaft und Raumplanung nicht decken, und auch hier steigt die Wahrscheinlichkeit, sich räumlich zu verpassen, mit der Komplexität der Inhalte, womit wiederum die höchsten Anspruchsund Anforderungsprioritäten besonders betroffen werden.

Die Kommunikationsbarriere, die auf dem räumlichen Verpassen beruht, wird zusätzlich durch die große Wahrscheinlichkeit verstärkt, daß der variierende Forschungsmaßstab nicht mit dem Problemmaßstab der gerade aktuellen raumplanerischen Aufgabe übereinstimmt.

\section{d. ... verpassen sich gesellschaftlich}

Eine der entscheidendsten Kommunikationsbarrieren zwischen Forschung und Raumplanung baut sich dadurch auf, daß die beteiligten Forscher und Raumplaner völlig anderen gesellschaftlichen Profilierungsumfeldern ausgesetzt sind. Das Profilierungsumfeld der Forscher ist die "Gesellschaft der Wissenschaften, deren Profilierungsanforderungen sich am Forschungsprozeß (vgl. oben) orientieren, während die
Planer ihr Profilierungsumfeld in der "Gesellschaft der Auftraggeber und Entscheidungsträgen sinden.

$\mathrm{Da}$ die Planer nicht zum Profilierungsumfeld der Forscher gehören - und umgekehrt -, ist die Kommunikationsintensität an sich schon gering. Weil der Planer in seinem "harten» Profilierungsumfeld inhaltlich und emotional standhaft sein muß, muß er möglichst vermeiden, sich von neuen, alte Standpunkte relativierenden Forschungsergebnissen verunsichern zu lassen. Damit baut er die Kommunikationsintensität weiter ab, v. a. auf Inhalten, die neue Methoden umfassen oder unverhoffte Erkenntnisse betreffen - doch gerade dies sind die Inhalte, die im Profilierungsumfeld der Forscher eine zentrale Stellung einnehmen.

\section{e. ...verpassen sich formal}

Auf der Ebene der formalen und sprachlichen Aufbereitung wissenschaftlicher Inhalte sind die Kommunikationsbarrieren zwischen Forschung und Raumplanung am offensichtlichsten, was dazu führt, daß hier die (gegenseitigen) Vorwürfe am häufigsten ansetzen. Bei näherer Betrachtung findet diese offensichtliche Kommunikationsbarriere ihre Begründung insbesondere im unterschiedlichen Suchprozeß und im unterschiedlichen Profilierungsumfeld (vgl. oben), weshalb auch hier besonders die komplexeren Inhalte, die gleichzeitig die höchsten Anspruchs- und Anforderungsprioritäten aufweisen, betroffen werden.

\section{Eine erste Bilanz zu den Auswirkungen der Kommunikationsbarrieren}

In der Figur sind die fünf erwähnten Ebenen, auf denen sich Forscher und Raumplaner verpassen und aufgrund deren sich Kommunikationsbarrieren aufbauen, zusammengestellt. Wenn anhand dieser Figur die Wirkung der kurz erläuterten Kommunikationshindernisse auf den Transfer der verschiedenen Kategorien von Inhalten im Sinne einer ersten Bilanz interpretiert wird, so zeigt sich ein recht deutliches Bild:

Der Transfer von dokumentarischen Inhalten wird am wenigsten durch Kommunikationsbarrieren behindert, und auch zu Erklärungsmustern einzelner (sektoraler) Sachverhalte kann ein gewisser Informationsfluß gewährleistet werden. Hingegen sind die Kommunikationskanäle für diejenigen Inhalte, die sowohl im Anspruch der Forscher als auch in den Anforderungen der Raumplaner einen hohen Stellenwert einnehmen, ziemlich verstopft. Es ergibt sich also die für beide Partner enttäuschende Situation, da $\beta$ Kommunikation bloß über Inhalte zustande kommt, die auf beiden Seiten von eher untergeordneter Bedeutung sind, womit die Gefahr, daß sowohl Forscher wie Planer das Interesse an einem Transfer verlieren, fast übermächtig wird. 


\section{Bemerkungen zu einigen Ansätzen zum Abbau der Kommunikationsbarrieren:}

In der beiliegenden Figur ist angedeutet, bei welchem der beiden Partner sich Möglichkeiten bzw. Grenzen beim Abbau der einzelnen Kommunikationsbarrieren ergeben. Aus diesen angedeuteten, thesenhaften Möglichkeiten und Grenzen seien hier zwei herausgegriffen:

Bei der Kommunikationsbarriere, die sich aufgrund des unterschiedlichen Suchprozesses aufbaut, kann der Raumplaner durch vermehrte (und optimistischere) beobachtende Begleitung des Forschungsprozesses einen Abbau der Kommunikationsbarriere bewirken, wobei hierbei der SGAG eine wichtige, unterstützende Funktion zukommen könnte. Hingegen muß die Wissenschaft die prinzipielle (iterative) Abfolge im Forschungsproze $B$ einhalten, um die beide Partner interessierenden, höchsten Anspruchsprioritäten sukzessive annähern zu können. Dabei können zwar vermehrt anwendungsorientierte Beispiele in diesen Forschungsproze $B$ einfließen, doch eine einseitige Ausrichtung auf derartige Beispiele würde den zentralen Forschungsproze $\beta$ und damit das beide Partner schlußendlich interessierende Forschungsziel gefährden.

Im Bereich der wichtigen Kommunikationsbarriere, die sich aufgrund der unterschiedlichen Profilierungsumfelder aufbaut, sind die Abbaumöglichkeiten ebenfalls beschränkt. Zwar sollte geographieintern die praktische Anwendung und Umsetzung von Forschungsergebnissen vermehrt honoriert werden, doch im Kontakt mit anderen Disziplinen sind hier deutliche Schranken gesetzt, indem sich die geographische Forschung im Disziplinenzirkus wissenschaftlich profilieren muß, um die Möglichkeiten zur interdisziplinären Zusammenarbeit, die für die Anspruchsrealisierung unabdingbar ist, aufrechtzuerhalten.

\section{Eine umformulierte These}

Aufgrund der Überlegungen zu den Kommunikationsbarrieren und den selektiv herausgehobenen Möglichkeiten und Grenzen zu deren Abbau läßt sich die These, die Ausgangspunkt dieses Beitrags war, neu formulieren:

Die Raumplaner geben sich zuwenig Rechenschaft darüber, daß die geographische Forschung die auch für die Raumplanung entscheidenden, langfristigen Aufgaben nicht mehr wahrnehmen kann, wenn sie sich nur noch von aktuellen, brisanten Problemen leiten läßt - was aber nicht heißt, daß sie es nicht in etwas verstärktem Maße tun dürfte.

Urs Wiesmann, Dr. phil. nat., Geograph, Geographisches Institut Universität Bern, 3012 Bern

\section{Die geographische Wissenschaft unterstützt die Raumplanung zu wenig}

Jürg Karlen:

Meine Überlegungen zu dieser These sollen als Diskussionsgrundlage verstanden werden, sie sind weder abgerundet noch vollständig. Das skizzierte Bild ist überspitzt ausgedrückt, wobei es mir nicht darum geht, die vorgegebene These zu bestätigen.

Bevor die Raumplanung an die geographische Wissenschaft Forderungen anmeldet, hat sie sich über ihre heutige Position im klaren zu sein. Die Raumplaner sind sich ihrer Verantwortung bezüglich der Erhaltung unseres Lebensraumes bewußt. Über alle politischen Parteien hinweg wird es als raumplanerische Aufgabe angesehen, dafür zu sorgen, daß unsere Umwelt vor Zerstörungen bewahrt bleibt. Alle miteinander tun wir uns äußerst schwer, ökologische Erkenntnisse und Forderungen in konkrete Handlungen umzusetzen. Woran liegt es, daß gerade in dieser Beziehung der Vollzug raumplanerischer Zielsetzungen nur sehr schwerfällig vor sich geht? Meiner Ansicht nach beginnt es schon damit, daß nicht jeder, der Ökologie sagt, das gleiche meint. Ökologie wird u. a. gleichgesetzt: mit qualitativem Wachstum, mit der Rückkehr zu selbstgenügsamen Lebensformen (Asketenvorstellungen und Verzichtsideologien), mit politischen Gruppierungen. Bereits aus diesen wenigen Beispielen des unterschiedlichen Ökologieverständnisses wird ersichtlich, wie schwierig es ist, ökologischen Handlungen zum Durchbruch zu verhelfen. Diese Tatsache wird durch das verlorengegangene Vertrauen in die nichtexakten Wissenschaften verstärkt. Keiner traut mehr den Resultaten und Aussagen des andern. Bald glauben wir nur noch derjenigen Statistik, die wir selber "gefälscht» haben.

Darf aus einer solchen Situation heraus die Raumplanung überhaupt an die geographische Wissenschaft Forderungen stellen? Die Geographie ist bestrebt, die Raumplanung $\mathrm{zu}$ unterstützen, ist aber angesichts dieser Unsicherheit die Art und Weise richtig? Persönlich glaube ich nämlich, daß die Geographie wesentlich mehr zur Lösung raumplanerischer Fragen beitragen könnte, wenn sie dies in einer Form täte, welche die Unsicherheit nicht noch vergrößert. Er ist nicht meine Absicht, zwischen der geographischen Forschung und der raumplanerischen Praxis eine Diskrepanz zu konstruieren. Ich meine nur, daß von seiten der Forschung die Einsicht wachsen sollte, daß die «Praxis» heute und nicht erst morgen - wenn auch mit unvollkommenem Wissen - Lösungen zu Umweltfragen aufzeigen muß. Ich weiß, sowohl hinsichtlich des ökologischen Erkenntnisstandes als auch in bezug auf das methodische Vorgehen in der ökologischen Planung bestehen noch Lücken. Es ist die Aufgabe der geographischen Forschung, diese Lücken weiter zu verringern sowie die ökologischen Erkenntnisse zu vertiefen. Es hilft der Praxis aber nicht weiter, 
wenn die geographische Wissenschaft vorwiegend auf diese Lücken hinweist, das trägt eben zur erwähnten weiteren Verunsicherung bei. Die Geographie verfügt doch heute über einen Wissensstand, der es ihr mit ein klein wenig «Mut zur Lücke» ermöglicht, klar und unmißverständlich Stellung $\mathrm{zu}$ beziehen $\mathrm{zu}$ den wesentlichen Umweltproblemen und zwar ohne Wenn und Aber. Das Risiko von Fehlinterpretationen schätze ich als gering ein. Die Geographie muß, um in der Planung ernst genommen zu werden, gegen außen weniger Zweifel verbreiten, sie darf auf ihren heutigen Erkenntnisstand stolz sein. Selbstverständlich hat die geographische Forschung parallel dazu weiterzugehen. Die Forschung soll an Aussagen zweifeln, sie darf sich durch Originalität auszeichnen, ohne aber der Meinung zu sein, daß alles "originelle» gleich in die Praxis umgesetzt werden muß. Für mich ist das Bestreben der Forschung, aus jeder Arbeit krampfhaft noch eine anwendungsorientierte Methode zu entwikkeln, falsch. Was soll der Praktiker mit komplizierten Methoden anfangen, über die der Forscher selber sagt: «Die Erfahrung hat gezeigt, daß das Verfahren relativ aufwendig ist. Es kann aber durch eine Reduktion der Kriterien praktikabler gemacht werden.» Dies sind - ohne den Wert der einzelnen Arbeit anzuzweifeln - unfertige Ergebnisse. Wer kauft einen Computer, von dem bekannt ist, daß er noch nicht läuft? Die Computerbranche unternimmt enorme Forschungsanstrengungen, die bleiben aber firmenintern bis jeweils wieder eine neue Computergeneration auf den Markt kommt. In der Geographie findet zwischen Forschung und Produkt eine ungute Vermischung statt, und letztlich sind Forscher wie Praktiker durch eine Flut unfertiger Resultate und Methoden nichts weiter als verunsichert. Ich fühle mich jedenfalls überfordert, all die gutgemeinten praxisorientierten Methoden 1. zu lesen, 2. ganz zu verstehen, 3. anzuwenden und 4. das Resultat noch einer Mehrheit der Bevölkerung klarzumachen. In dieser Beziehung erwarte ich eine Unterstützung von der Geographie, indem sie wieder einmal eine klare Trennung zwischen Forschungsergebnissen und Produkten (= geographische Wissenschaft) vornimmt und nur mit fertigen Produkten an die breite Öffentlichkeit tritt. Das gäbe wieder mehr Sicherheit, die Erkenntnisse bei der Lösung von Umweltproblemen anzuwenden, die Bevölkerung davon $\mathrm{zu}$ überzeugen und die erforderlichen Maßnahmen politisch durchzusetzen. Die geographische Wissenschaft als Bindeglied zwischen Forschern und Praktikern. Beide könnten so wieder überzeugter auftreten, die anstehenden Probleme im eigenen Aufgabenbereich lösen und nicht mit viel Aufwand sich gegenseitig neue Probleme schaffen. In einer solchen Form würde die geographische Wissenschaft zu einer wertvollen Stütze der Praxis.

Mit meiner Aufforderung zur vermehrten Unterstützung der Raumplanung durch «Vereinfachungen» spreche ich vornehmlich die geographischen Hoch- schulinstitute an. Sie alle vertreten miteinander die geographische Wissenschaft. Die Praxis hat ebenfalls ihren Beitrag zu leisten. Ein erster Schritt in die richtige Richtung besteht für mich in der Förderung der Zusammenarbeit (gemeinsame Projektbearbeitungen) sowie im Austausch einzelner Geographen zwischen Hochschulinstituten und Planungsämtern oder -büros für eine gewisse Zeit. Das gegenseitige Verständnis ist zu verbessern, verfolgen wir doch ein gemeinsames Ziel - die Erhaltung unserer Umwelt.

Jürg Karlen, dipl. Geograph ETH, Raumplaner, Stadtrat, 5000 Aarau

\section{Ökologische Methoden in der Raumplanung}

Methoden zur Berücksichtigung ökologischer Belange sind vorhanden, aber sie werden von der Raumplanung nicht übernommen und angewendet, obwohl die rechtliche Basis vorhanden wäre

Hartmut Leser:

\section{Kritische Betrachtungen zur Berücksichtigung und Verwendung ökologischer Belange in der Raumplanung}

\section{Der Vorwurf an die Ökologen}

Sowohl die Raumplanung als auch andere Anwenderbereiche "der Praxis» erheben immer wieder den Vorwurf, die Ökologie-Wissenschaften würden an den Bedürfnissen «den» Praktiker vorbeiforschen und/oder Material bereitstellen, das für eine Umsetzung und Verwendung in der Praxis ungeeignet sei. Dabei wird übersehen, daß in der Ökologie seit Jahren eine andere Situation herrscht. Das heißt, der Praktiker mißt die Ökologiewissenschaften immer noch an den desolaten Verhältnissen, die er während seiner Ausbildung erlebt hat. Es wäre an der Zeit, umzudenken.

\section{Methodische Problemaspekte, die im Vorwurf stecken}

Prämisse ist, daß die ökologisch forschenden Disziplinen aus dem Geo- und Biobereich - je nach Interessensrichtung der jeweiligen Institution - mehr oder weniger praktisch verwertbare Ergebnisse bereitstellen. Sie haben das bewiesen, wenn sie selbst im Anwenderbereich tätig sein durften. Bei diesen Erfahrungen tauchten einige methodische Grundaspekte auf, die vom Vorwurf der Praktiker unberücksichtigt gelassen werden:

- «Die» Praxis gibt es nicht. Sie kann demzufolge auch nicht pauschal mit Forschungsergebnissen versorgt werden. 
* Es gibt hingegen in der Praxis Anwenderinteressen, die von vielen Einzelproblemen repräsentiert werden. Diesen Einzelproblemen kann man nur ganz gezielt zuarbeiten. Dazu gehört ein Auftrag. Das Instrumentarium steht bereit, z. B. ökologische Analysen für Kiesgrubenrekultivierungen, Gebietswasserhaushaltsbestimmungen, Stadtklimakennzeichnungen usw., vorzunehmen.

- Die Einzelprobleme weisen unterschiedliche Größenordnungen auf, die ganz verschiedene Methodiken und Maßstäbe bei ihrer planungspraktischen Bewältigung erfordern.

* Dem Praktiker ist dies oft nicht bewußt. Er versäumt, beim Ökologen nachzufragen und arbeitet mit hausgemachtem Material, das dem praktischen Gegenstand nicht adäquat ist. Die ökologischen Aussagen, die zur Bewältigung dieser Einzelprobleme erforderlich sind, können sich im Maßstab zwischen 1:100 und 1:25000 bewegen. Sie haben dann jeweils andere inhaltliche Gewichtungen und damit unterschiedliche Aussagewerte. Parzellenscharfe Aussagen sind dem Ökologen möglich, aber der Praktiker muß sagen, ob sie immer nötig sind.

- Die Forschungsergebnisse der ökologischen Fachwissenschaften werden nicht immer im Hinblick auf eine Multiaussage erarbeitet, sondern sie verfolgen in der Regel disziplininterne Ziele. Daraus resultiert aber noch lange nicht, daß diese Ergebnisse nicht anwendbar seien.

* Ein Teil der Ergebnisse ist (Übereinstimmung des Maßstabes der Untersuchungsergebnisse und des Bedürfnisses des Anwenders und damit des praktischen Problems vorausgesetzt) fast immer direkt anwendbar. Dies ergibt sich aus der heute allgemein üblichen großmaßstäbigen (topischen) Arbeit und aus dem systemtheoretischen integrativen Ansatz, der die Probleme in ihrem landschaftlichen Kontext erfaßt. Andererseits ist es aber auch möglich, einen Auftrag aus der anwendenden Praxis ganz gezielt zu erfüllen, wenn dafür präzis die Randbedingungen genannt werden. Die erstgenannten Ergebnisse lassen sich zudem mit Hilfe des Fachwissenschaftlers in eine praktisch verwertbare Form umsetzen.

- Fachökologische Ergebnisse sind unverständlich und entziehen sich somit einer Beurteilung und damit auch Weiterverwendung durch den anwendenden Praktiker.

* Es wird übersehen, daß nicht nur die Raumplanungsgeneralisten, sondern auch die Fachwissenschaften ihre Fachsprachen haben, die der disziplinären Verständigung dienen. Ebenfalls wird übersehen, daß sich in den Ökowissenschaften in den letzten zehn bis fünfzehn Jahren ein fachübergreifendes Vokabular herausgebildet hat. Der anwendende Praktiker ist gehalten, sich mit diesem Vokabular auseinanderzusetzen und die Entwicklungen im Ökosektor zu verfolgen. Er kann dabei auf die Hilfe der Ökologen zählen.

\section{Das Grundproblem "ökologische Belange in der Raumplanung und Praxis»}

Bewußt werden hier Details ausgespart und das planerisch-praktische Problem auf «Nutzungsplanungen» (unabhängig von deren Art, Umfang und Maßstab) zusammengezogen. Dem Ökologen fällt dabei folgendes auf:

- Fachplanungen sind offensichtlich in der anwendenden Praxis die Norm. Sie gehen möglicherweise auf nicht mehr zeitgerechte administrative Strukturen zurück, die zu ändern wären.

- Auch innerhalb und zwischen den Fachplanungen läßt sich integrativ und nicht nur separativ arbeiten. Trotzdem scheint das sektorale Vorgehen der Anwender die Regel zu sein. Daher kann er auch mühelos und ohne Gewissensbisse auf ökologischintegrative Grundlagen seiner Arbeit verzichten. Er ist "nicht zuständig» für Zusammenhänge der Art «Natur/Technik/Gesellschaft».

- Geplant und gewirtschaftet wird immer noch, als seien Boden, Klima, Wasser, Georelief und Biosphäre vermehrbare Güter und als würden sie in den Agglomerationen (und sonstigen Überbauungsgebieten) keine Rolle spielen. Das ist eine grundlegende Verkennung eines Naturgesetzes: Ökosysteme sind überall, und sie funktionieren auch überall naturgesetzlich, selbst wenn sie anthropogen verändert sind. Dieser Grundtatsache wird seitens der Praktiker nicht Rechnung getragen.

- Umweltprobleme, und somit ökologische Sachverhalte in der Raumplanung, sind komplexe Sachverhalte, deren Wirken und Funktionieren sich großmaßstäbig abspielt. Durch das Gesetz von den Ökosystemhierarchien in der landschaftlichen Realität (dazu gehört auch die Agglomeration) ergeben sich natürlich auch mittelmaßstäbige Zusammenhänge mit zum Teil kleinmaßstäbigen Auswirkungen (z. B. Zusammentreten von Mikroklimaeffekten zu Mesoklimawirkungen usw.). Dieser Komplexität muß vom Ansatz und der Methodik der Planung her Rechnung getragen werden.

- Praktiker stellen an den Ökologen gelegentlich die falsche Frage: "Wie weit kann ein Raum belastet werden?». Dabei wird übersehen, daß komplexe Belastungen nicht genau bestimmbar sind und die Weiterentwicklung der Ökosysteme nur an Hand von Indizien abgeschätzt werden kann. Planerisches Prophetentum liegt dem Naturwissenschaftler nicht. Letztlich ist auch das komplexe Funktionieren der Ökosysteme nur quantitativ abschätzbar, weil ein zu erforschendes Ökosystem immer nur ein Modell der Wirklichkeit sein kann. 
Ohne die Reihe der Problemaspekte verlängern zu wollen, soll schon hier zusammengefaßt festgestellt werden, daß es dem anwendenden Praktiker offenbar schwerfällt, sich mit den Grundlegungen, Regeln und Gesetzmäßigkeiten moderner ökologischer Forschungen vertraut zu machen. Er übersieht, daß die Norm für den zu planenden Raum, auch wenn er durch neue Nutzungen verändert werden soll, das funktionierende Ökosystem ist, auf/in/an dem sich alle Nutzungen abspielen (sollen). Statt dessen arbeitet er - sektoral, d. h. in Fachplanungen,

- in Maßstäben, die der ökologischen Realität, d.h. dem komplexen Funktionieren der Ökosystemen, nicht Rechnung tragen,

- zu hastig, weil er sich vermeintlich unter politischem und administrativem Druck sieht und somit ökologische Grundlagen nicht berücksichtigen «kann», die einen gewissen Bearbeitungsaufwand erfordern.

Man muß sich sowohl in der Nutzungsplanung, welcher Art auch immer, als auch in Verwaltung und Politik allmählich mit dem wirklichen Sachzwang anfreunden, daß bei ökologischen Gebietsbestimmungen, sollen sie seriöse Planungsgrundlagen bilden, keine "Schnellschüsse» möglich sind. Der Einsatz oder Nichteinsatz seriöser ökologischer Grundlagen in der Planung scheint, angesichts der Langsamkeit der Planungs- und Entscheidungsmühle, auch eher ein organisatorisches Problem zu sein:

- Der Raumplaner muß wissen, daß seine Nutzungsplanungen über eine ökologische Grundlegung verfügen müssen (d.h. er muß das Problem überhaupt erkennen und einordnen und damit dem Ökologen einen sachlich richtigen Auftrag zur Erarbeitung einer ökologischen Gebietsanalyse erteilen können), und:

- dieser Auftrag wäre in dem nun wahrhaft langen Planungs- und Entscheidungsprozeß so rechtzeitig zu erteilen, daß dem Ökologen genügend Zeit für eine saubere Analyse bleibt.

Das setzt aber voraus, daß der Raumplaner einmal über ausreichende landschaftsökologische Grundkenntnisse verfügt und zum anderen auch gewillt ist, den Grundgedanken von der Funktion des Lebensund Wirtschaftsraumes des Menschen als eines Ökosystems in seine Planungsmethodik und -konzeptionen einzusetzen.

\section{Das Problem der "methodischen Hintertür» in der Raumplanung}

Die Raumplanung sieht sich in einem Dilemma: Einerseits möchte man ökologische Grundlagen welcher Art und wie auch immer - mitberücksichtigen, andererseits kann und möchte man sie nicht selbst erarbeiten. Eine scheinbare Auflösung dieses Dilemmas bot sich mit den verschiedensten "Landschaftsbewertungsverfahren» an, die z.T. nur mit neuen Etiketten versehen wurden und dann als «Ökologische Risikoanalyse», «Umweltverträglichkeitsprüfung» oder «Environmental Impact Analysis» die seriöse ökologische Gebietsanalyse umgehen.

Sicherlich tut man dem einen oder anderen Verfahren, deren Tauglichkeit am richtigen Platz der Methodik und zum rechten Zeitpunkt im Planungsproze $ß$ unbestritten ist, mit einer pauschalen Ablehnung Unrecht. Trotzdem wäre festzustellen, daß die meisten Bewertungsverfahren, wenn sie als Ersatz für ökologische Gebietsanalysen eingesetzt werden, als gefährlich bezeichnet werden müssen:

- Sie aggregieren willkürlich zahlreiche Einzelgrößen, die irgendwie an der Landschaftsrealität beteiligt sind, deren Stellung im Ökosystem aber unklar ist.

- Sie verwenden landschaftsökologisch irrelevante Parameter, die allenfalls "schmückende Funktion» haben, jedoch keine maßstabsbezogene Aussage über das Ökosystem liefern.

- In der Regel werden lineare Abhängigkeiten bewertet, nicht jedoch Funktionsnetze, wie sie für die Ökosysteme kleiner oder großer Dimensionen charakteristisch sind.

- Die Bewertungen sind "Schnellschüsse», d.h. sie werden rasch erstellt - unter Verwendung von Sekundärmaterial. Primärdaten und -fakten sind selten.

$\mathrm{Da} ß$ diese hier nur grob skizzierten Schwächen auch von den anwendenden Praktikern selbst erkannt werden, läßt sich aus der Fülle der Verfahren und ihren Modifikationen ableiten. Offensichtlich gibt es keine Möglichkeit, eine quasi-intersubjektive planerisch-«ökologische» Gebietskennzeichnung vorzunehmen, die den Vergleich mit einer naturwissenschaftlich begründeten und in der topischen Größenordnung quantitativ-exakten landschaftsökologischen Gebietsanalyse standhält. Statt dessen wird durch permanente Kosmetik an den Verfahren versucht, diese Schwächen zu kaschieren. Planungen können bekanntlich nicht besser als ihre Grundlagen sein. Wenn also von Bewertungsverfahren zweifelhafter Art ausgegangen wird, statt die Möglichkeiten der zur Verfügung stehenden ökologischen Gebietsanalysen wahrzunehmen, dann muß mit Planungseffekten gerechnet werden, die zur «Zufallslandschaft» führen. Sie ist zwar geplant, aber sie funktioniert nicht. Sie wird vom Bewohner als unwirtlich empfunden. Mit ihr kann er sich nicht mehr identifizieren. Diese methodische Hintertür, die in der Raumplanung den Normalfall darstellt, kann weder vom Ökologen noch vom von der Planung betroffenen Bewohner des Planungsraumes akzeptiert werden.

Es geht nicht darum, die Bewertungsverfahren generell zu verdammen. Sie erfüllen aber ihren Nutzen erst dann und vermeiden damit zugleich ihre fundamentalen Schwächen auf geographisch-landschaftsökologisch-ökofunktionalem Bereich, wenn sie an der richtigen Stelle eingesetzt werden. Dazu gehört zunächst einmal, daß der anwendende Praktiker 
gedanklich akzeptiert, daß sein Planungsgegenstand (auch bei einer noch so banalen Nutzungsplanung) immer noch das Ökosystem ist - auch und gerade in der Agglomeration, wo tiefgreifende Änderungen in den natürlichen Ressourcen erfolgt sind - und gleichwohl mit diesen Ressourcen zu wirtschaften und zu leben ist. Daraus resultiert folgender Ablauf des Vorgehens am Gegenstand.

\section{Raumbewertungsverfahren und ökologische Gebiets- analyse - keine Alternativen!}

Die ökologische Gebietsanalyse und die Raumbewertungsverfahren nehmen als Arbeitsschritte in der anwendenden Praxis ganz bestimmte Plätze ein.

\section{Planer}

Projektbeschreibungen/Problemerkennungen/Auftragserteilung.

\section{Ökologe}

Ökologische Gebietsanalyse

Vorgehen: Integrativ mit komplexer Methodik.

Gegenstand: Realer Raum (mit oder ohne anthropogene Veränderungen).

Maßstab: Entsprechend dem planerischen Anforderungsprofil, aber überwiegend großmaßstäbig, d. h. in topischer Dimension, weil nur dann die Ergebnisse und Aussagen auf den konkreten Gegenstand Bezug nehmen und zugleich quantitativ sein können.

Ergebnis: Ökologische Gebietsfunktionstypen (mit der Möglichkeit des Auszugs von analytischen Grundlagenfakten und Einzelfaktorenbeschreibungen).

\section{Planer}

Bewertung $I=$ Abschätzen des Naturraumpotentials durch Bewertungsverfahren im Hinblick auf Nutzungen.

Bewertung II: Abwägen der Nutzungen/Neunutzungen untereinander durch Bewertungsverfahren.

Der Planer legt anschließend seine Ergebnisse dem politischen Entscheider vor. Die Verantwortung und Verantwortlichkeit des Planers ist groß, weil er seine Bewertungen an gesellschaftlichen, politischen und ökonomischen Normen und Setzungen ausrichten muß. Diese Normen sind bekanntlich fließend. Die ökologischen Fachwissenschaften sind sich jedoch darin einig, daß Entscheidungen realitätsbezogener ausfallen und auf die Normen rückkoppeln können, wenn sie auf ökologischen Gebietsanalysen basieren. Aus der obigen Abfolge geht weiterhin hervor, daß die ökologische Gebietsanalyse eine an sich unverzichtbare Basis planerischer Arbeit und Entscheidungen darstellt, deren Bedeutung für die Qualitätssteigerung der planerischen Aussage bis heute in der anwendenden Praxis noch nicht erkannt ist.

Hartmut Leser, Prof. Dr., Forschungsgruppe Physiogeographie und Geoökologie, Geogr. Institut Universität Basel, Klingelbergstr. 16, 4056 Basel

\section{Der Raumplanung fehlen wissenschaftlich fundierte und praktisch abgesicherte Methoden zur Konflikt- bewältigung}

Hans-Dietmar Koeppel:

Im Vordergrund meiner Überlegungen steht die Frage, ob es eine Konfliktbewältigung bei der Berücksichtigung ökologischer Belange überhaupt geben kann. Daran anknüpfend gilt es der Frage nachzugehen, wann ein Konflikt bei der Berücksichtigung ökologischer Belange als bewältigt gelten darf (und auch wer darüber zu befinden hat). Und schließlich dann stellt sich die Frage nach Methoden, deren Brauchbarkeit und deren Notwendigkeit.

\section{Eine Konfliktbewältigung bei der Berücksichtigung ökologischer Belange kann es im eigentlichen Sinne nicht geben.}

Hier muß man sich fragen, welcher Art sind denn die Konflikte, zwischen welchen Sachverhalten resp. Interessenlagen bestehen oder entstehen Konflikte?

- Verstehen wir unter ökologischen Belangen alle Abläufe und Zustände in unserer Natur und Landschaft, so gilt hier die obige These schon deshalb, weil die Konflikte zwischen den «ökologischen Interessenlagen» geradezu die Abläufe in Natur und Landschaft ausmachen. Ein Ausräumen der Konflikte würde einem Abschalten des Motors gleichkommen. In diesen Abläufen passiert doch ein ständiges Verlieren und Gewinnen, ein Wachsen und Schwinden, ein Überhandnehmen und ein beinahes oder völliges Verschwinden. (Der Stellung des Menschen kommt hier eine Konfliktlösung nicht zu).

- Andersartig ist die Konfliktsituation zwischen den Abläufen und Situation in Natur und Landschaft und den Interessen der Menschen, welche diese Abläufe und Situationen oder anders die Begabungen und Eignungen eines Raumes (mehr oder weniger gewinnbringend) nutzen wollen. Hier besteht der Konflikt in der Begrenztheit und Endlichkeit der Leistungen oder in einer (passiven) Leistungsverweigerung. Ein Ton- oder Kiesvorkommen ist ebenso begrenzt wie die Fläche als solche, wie die Aufnahmefähigkeit von Giften oder wie die Verkraftung von Beschädigungen, Belästigungen und Störungen verschiedenster Art. Das heißt doch nichts anderes, als daß die Wesensart des einen Konfliktpartners demnach eine Konfliktbewältigung als solche gar nicht zuläßt. 
In diese Konfliktart spielt die Raumplanung hinein. Sie kann reagieren z. B. mit Konzepten zur mittelund langfristigen Sicherung von Ressourcen. Sie kann sich also um den Stil der Bewirtschaftung kümmern. Methoden sind nicht gefordert.

- Die dritte Konfliktart besteht allein zwischen den o.g. Interessenlagen des Menschen, die bei der Nutzung der Begabungen und Eignungen eines Raumes mehr oder weniger deutlich und schnell miteinander in Konkurrenz geraten. Bedingt ist dies zu einem guten Teil von der o.g. Endlichkeit der natürlichen Lebensgrundlagen. Konfliktbewältigungen, die hier erforderlich sind, dienen jedoch nicht der Berücksichtigung ökologischer Belange, sondern dem Verteilungskampf um die Begabungen und Eignungen eines Raumes resp. um diesen als solchen. $\mathrm{Da} \beta$ in die Methoden zur Bewältigung dieser Konfliktart heute zunehmend Argumentationen einfließen, die eine Berücksichtigung ökologischer Belange beinhalten, ist in letzter Konsequenz Mittel zum Zweck und daher Augenwischerei.

Beispiele wie die NHT oder die Hochrhein- und Aareschiffahrt, die angeblich projektimmanent ökologische Belange stärker berücksichtigen als eine Nationalstraße, mögen dies verdeutlichen. Beide verändern massiv. Anders sähe das aus, wenn gleichzeitig mit einem solchen Projekt der Rückbau eines anderen Verkehrsträgers gekoppelt wäre, d. h. die Planung NHT ist gekoppelt mit der Aufhebung der N1, um bei diesem Beispiel zu bleiben.

Die Raumplanung und ihre Methoden spielen für diese Konfliktart eine große Rolle. Der Raumplanung wird zugebilligt, daß sie sich ehrlich um eine Berücksichtigung ökologischer Belange bemüht, nicht aber ihren Partnern, zwischen denen sie zu vermitteln hat. Hier dient die Planung als solche bereits zu einem guten Stück der Konfliktbewältigung.

\section{Die Entscheidung über eine erfolgreiche Konflikt- bewältigung kann erst auf der Ebene der Realisierung und einer zeitlich mehr oder weniger davon abgesetzten Erfolgskontrolle stattfinden.}

Dazu ein einfaches Beispiel: Ein Amphibien-Laichgewässer soll infolge einer Straßenbaumaßnahme überdeckt werden. Der Konflikt wird gelöst durch die Projektierung eines neuen Laichgewässers neben dem Trassée in guter Zuordnung zum Jahreslebensraum der Population.

Problem 1:

Das alte Laichgewässer wird überdeckt, bevor das neue Laichgewässer gebaut werden konnte. Die Population verliert eine Generation. Das aufgrund eines hohen Grundwasserstandes nicht abgedichtete Gewässer bleibt wegen mangelnder Frühjahrsniederschläge 1983, 84, 85 trocken, nachdem es im Spätsommer 1982 bei Erstellung randvoll war. Die Population verliert bisher 4 Generationen. Für ein Überleben einer kleinen Restpopulation kann keine Garantie gegeben werden.

Es wird hier niemand bestreiten, daß die Konfliktlösung im Projektstadium erfolgreich war. Die Realität zeigt ein gegenteiliges Ergebnis.

\section{Eine Entscheidung in der Konfliktlösung für einen zu} erhaltenden oder angestrebten Zustand schließt jedesmal einen anderen möglichen Zustand aus, der auch Werte hat.

Auch diese Aussage, die als ein Fazit aus den beiden vorgenannten gezogen werden kann, soll ein Beispiel erläutern:

Die Schaffung von Kiesgruben wurde bis vor knapp 10 Jahren von Natur- und Landschaftsschutz bekämpft. Innerhalb weniger Jahre sind auf Naturschutzseite die enormen Werte der Kiesgruben als Lebensraum in unserer sonst so künstlich stabil gehaltenen Landschaft erkannt worden. Man fährt heute gern auf dem Züglein Kiesgrube mit, sofern einige Teilflächen diese Lebensraumqualitäten tragen dürfen.

Wertverschiebungen, die unbedingt zulässig sein müssen, lassen also eine erfolgreich scheinende Konfliktlösung in das Gegenteil umschlagen. Läßt sich dann überhaupt noch von Konfliktlösungen sprechen?

\section{Die Methoden zur Berücksichtigung ökologischer Belange sind oberhalb der Realisierungsebene angesie- delt. Sie dienen daher nicht der Konfliktbewältigung, leisten aber wichtige Beiträge zum Erkennen von Kon- flikten und zum Herleiten der jeweils relativ umwelt- freundlichsten Lösungen.}

\section{Der Stellenwert dieser Methoden als reines Hilfsmittel muß stets im Bewußtsein des Anwenders bleiben.}

Die erforderliche Exaktheit und Komplexität der Methoden führt in der Anwendung zur Aufspaltung von Sachverhalten. Der Blick für den ganzen Sachverhalt wird abgelenkt, die Beurteilung bezieht sich dann mehr auf die Frage des richtigen Umgangs mit den Einzeldaten. Das führt weiter dazu, daß Ergebnisse akzeptiert werden, weil die Methode und der Umgang mit Daten stimmig sind, ohne daß noch begriffen wird, was hinter den Zahlen für ein Sachverhalt steht. 
6. Die in der Regel aus der Ökonomie stammenden Methoden mit Bilanzierungsansätzen usw. reichen nicht für Sachverhalte ökologischen Inhalts und schon gar nicht für Fragen vom Landschaftsschutz.

Bei der Anwendung der Methoden vergessen wir schnell die Realität bei und nach der Realisierung (die für mich immer noch Raumplanung ist). Sie täuschen uns ein intellektuelles Verkraften und Bewältigen vor. Sie verführen daneben zu Manipulationen mit Daten.

\section{Die Entwicklung und Anwendung von Erklärungs- modellen und daraus abgeleiteten Methoden waren und bleiben nicht unnütz. Die Verknüpfung der Erklärungs- modelle zu einem Ganzen ist schwer und gelingt meistens nicht.}

Die Begründung sehe ich darin, daß ein Spezialist, der in der Lage ist, schlüssige Teilmodelle von Wirkungsketten zu entwickeln, nicht ausreichend Generalist bleibt, um sein Spezialwissen und die daraus abgeleiteten Zielvorstellungen noch richtig und maßvoll in eine Gesamtschau zu stellen. Wir brauchen aber Spezialisten, die auf die Ganzheit einwirken und sich einordnen können.

Andernfalls bleiben wir in der Situation, daß bei Konflikten der siegt, der am lautesten schreit, und nicht der, der recht hat. Das heißt, daß die Tagesform entscheidend sein kann.

Die Hochschule ist hier sicher gefordert unter Anerkennung, daß es diese Situationen gibt, Methoden zu entwickeln, die Spezialwissen mit dem jeweils richtigen Stellenwert zu einer Gesamtbeurteilung zusammenfließen lassen.

\section{Die derzeitigen Methoden zur Berücksichtigung ökologischer Belange führen zu Ergebnissen, die als Entscheidungsgrundlagen dem Politiker weitergegeben werden. Das Feld der Entscheidung wird damit dem Politiker überlassen. Das ist bequem, aber falsch!}

Die Entscheidung, wann ökologische Belange ausreichend berücksichtigt sind, ist Sache der Wissenschaft und nicht der Tagespolitik (wie die Ökonomie).

\section{Die Berücksichtigung ökologischer Belange in der Raumplanung benötigen eine kontinuierliche Forschung.}

Verfehlt sind auf der einen Seite Superforschungen à la $\mathrm{MAB}$, wie auf der anderen die Abkehr von der Wissenschaft hin zum Rauchen von Friedenspfeifen. Besondere Aufmerksamkeit und Kontinuität verdient die Erfolgskontrolle für das raumplanerische Handeln schlechthin. Die heutige Arbeitsteilung entzieht uns die Möglichkeit, unsere Projektideen selbst zu realisieren, ihre fortlaufenden Auswirkungen zu überwachen. Es mangelt uns in der Folge, den Erfahrungen im weiteren raumplanerischen Handeln den erforderlichen Stellenwert zu geben. Ein eklatanter Mangel wird das bei der Berücksichtigung ökologischer Belange.

Neben der Arbeitsteiligkeit verhindert auch der schnelle Wechsel beeinflussender Größen ein ausreichendes Sammeln von Erfahrungen.

10. Die Berücksichtigung ökologischer Belange erfordert eine Abkehr von einem finalen Planungsverständnis (siehe 1.). Benötigt werden hierfür Methoden einer Zielannäherung, welche nachfolgenden Generationen Entscheidungsspielräume offen lassen und sich nicht mit sogenannten Sachzwängen über Gebühr konfrontieren. (Großtechnologie macht das aber mehr als Kleintechnologie).

Auf den Ausgang zurückkommend heißt dies, daß wir uns darein schicken sollten, mit unbewältigten Konflikten zu leben, daß wir aber alles daran setzen sollten, den ökologischen Belangen mehr Stellenwert zuzubilligen, indem wir uns bereit zeigen, Entscheide zu fällen und zu tragen, auch wenn gewisse Unsicherheiten bestehen.

11. Wenn Methoden zur Konfliktbewältigung wirklich da wären, ginge uns Menschen gleichzeitig viel verloren. Denn auch die reine Ökologie kennt in ihren Werterhaltungen nicht alles, was uns Menschen erfreut, Bewunderung abringt.

Emotionen und damit verbundene Wertsetzungen beim Feststellen von einmaligen Sachverhalten, das innere Erregtsein bei der Betrachtung einer Landschaft in einem außerordentlichen Licht oder beim Erkennen eines besonderen Symbolgehaltes eines Ortes. All dies dürfte keine Entscheidungen mehr mit beeinflussen.

So wie wir der künftigen Generation Spielräume für eigene Wertschätzungen lassen sollten, so sollten wir diese auch für uns beanspruchen: Schönes empfinden zu können, ohne sofort den ökologischen Wert dieser Empfindung für unsere persönliche Regeneration zu analysieren und zu bewerten. Denken wir darüber nach!

Hans-Dietmar Koeppel, dipl. Ing., Landschaftplaner, Metron AG, Steinackerstr. 7, 5200 Windisch 


\section{Schlussbetrachtung}

Gilbert Thélin:

Es besteht die Absicht, am Geographentag vom 2./3. Mai 1986 ein Leitbild "Geographie Schweiz» zu entwerfen und zu verabschieden. Angesichts anstehender und ungelöster Umweltprobleme sowie Probleme auf dem Berufsfeld soll dieses Leitbild dazu dienen, unserem Fach neue Impulse für eine professionellere Organisation und Struktur zu verleihen.

Themenauswahl und thematisch akzentsetzende Ausgangsfragen haben auf dem Appenberg zu angeregten und umfassenden Diskussionen geführt, welche ganz in der Zielrichtung des Geographentages liegen. Es ist die feste Absicht der SGAG, die dort von den Referenten und Diskussionsteilnehmern entwickelten Ideen und konkreten Vorschläge zur Stärkung der Rolle der Geographie in der Praxis anläßlich der kommenden Leitbilddiskussionen $\mathrm{zu}$ vertreten und damit den Schritt in die praktische Umsetzung einzuleiten

Die Vorschläge der Appenbergtagung können wie folgt zusammengefaßt werden:

\section{Wünsche an die Forschung}

Von der Forschung erwarten die Praktiker die Kompilation praxisrelevanter Forschungsresultate sowie die Behandlung praxisbezogener Fragestellungen in den Bereichen Raumbeobachtung, Raumplanung und Umweltverträglichkeitsbewertung.

\section{Wünsche an die Ausbildung}

Angeregt wird ein dreistufiger Lehrplan mit Grundstudium, Fachstudium und integrativem Geographiestudium sowie eine praxisbezogenere, marktgerech- tere Ausbildung und ein «Lehrstuhl» für praktische Umsetzung.

\section{Forschungspolitik}

Unter den Praktikern herrscht die Meinung, daß sich die Geographie trotz größer werdenden Konkurrenz nicht aus der Raumplanung resp. Raumforschung zurückziehen darf; Themen wie «Die Landschaft als Objekt der Richt- und Nutzungsplanung» oder «Die Landschaft als Objekt der UVP» müssen der Geographie erhalten bleiben. Das Forschungsfeld ist eindeutiger abzustecken und innerhalb der Schweiz besser zu strukturieren. Auf forschungspolitischer Ebene muß sie sich mehr Einfluß verschaffen.

\section{Verbandstätigkeit}

Vorgeschlagen wird die Schaffung eines Verbandssekretariates für Dokumentation, Studienberatung, Veranstaltungskoordination, Vernehmlassungen zu politischen Fragestellungen, Stellenvermittlung, Weiterbildung, Information usw.

Auch die Ausarbeitung von Handbüchern mit den wichtigsten praxisorientierten Methoden und Verfahren oder mit den Adressen von Gesellschaften, Behörden, Öko- und Geographenbüros sowie Listen aktueller Forschungsprojekte wird zur Diskussion gestellt.

Zur Verbesserung der Weiterbildung der Berufstätigen wird die Schaffung eines gesamtschweizerischen Weiterbildungskurses, die Zusammenarbeit mit anderen Disziplinen, die Schaffung einer Verbandszeitschrift (Ausbau der GH?) sowie der Einsatz von Universitätsassistenten in der Praxis angeregt.

Gilbert Thélin, Dr. phil. nat., Präsident der SGAG, Lindenrain 8, 8038 Kirchlindach 